and unintended outcomes depending on features of the healthcare and income support system, as well as other contextual factors such as socioeconomic status and labour force composition.

Conclusion Income support systems impact the healthcare quality and functional capacity of people with work disability and LBP through context-dependent financial control, regulatory, and administrative mechanisms. Future policy design and research efforts should consider how income support systems may indirectly influence workers with LBP via the workplace.

\section{O4B.4 PREDICTORS OF RETURN TO WORK WITH READAPTATION IN PUBLIC WORKERS}

\begin{abstract}
'Adriano Dias*, 'João Marcos Bernardes Bernardes*, 'Sandro Augusto Servilha Coquemala, ${ }^{2} J u a n$ Gómez-Salgado, ${ }^{2}$ Carlos Ruiz-Frutos. ${ }^{1}$ Botucatu Medical School/Paulista State University, Botucatu, Brazil; ${ }^{2}$ University of Huelva, Huelva, Spain
\end{abstract}

\subsection{6/OEM-2019-EPI.94}

Objectives To identify factors associated with work readaptation after sick leaves in a group of public workers in Brazil.

Methods Case-control study of sick leaves in a university campus from 2010 to 2015. Logistic regression models were adjusted for two different response variables: readaptation and readaptation with limitations. A digital database was created and completed with data from manual sources.

Results Age at the beginning of the process, number of sick leaves, those of more than 16 days, average duration (total time of sick leaves/number of medical records), and mid-level healthcare positions were associated with work readaptation. In the model of readaptation with limitations, the age of hiring by the university, the number of sick leaves, those of more than 16 days, and mid-level healthcare positions, both rural work and operational positions were associated to the response variable.

Discussion A computerized database has been created, based on manual records, which has allowed us to identify labour and non-labour factors associated with the return to work after a sick leave and the possible functional readaptation, with or without limitation, in public workers. This has allowed proposing more efficient management of health measures to this population.

\section{O4B.5 MEASURING ASSOCIATION OF OCCUPATIONAL LIGHT VEHICLE DRIVING WITH LOW BACK PAIN: AN IRT APPROACH}

Michel Grzebyk*, Stéphanie Boini, Mathieu Dziurla, Jean-Jacques Atain- Kouadio, Anca Radauceanu. Inrs, Vandoeuvre Lès Nancy, France

\subsection{6/OEM-2019-EPI.95}

Low back pain (LBP) is a common problem for drivers but the analysis of associations of occupational exposures with LBP is hampered by the absence of a standardized clinical examination. Therefore, LBP is commonly assessed by a questionnaire in which useful information is scattered over many items. Many one-item-at-a-time analyses may not adequately evaluate these associations. We applied item response theory-like model (IRT) with one latent variable to examine the association of occupational light vehicle driving with LBP in 406 workers in the mail and parcel delivery sector using a 14-items questionnaire on LBP in the last 12 months investigating the cumulative duration of LBP, pain intensity, level and radiation in the lower limbs, medical consultation, pain medication use and sick leaves. We normalized the latent variable by constraining its variance to one. The link between the latent variable and the LBP indicators was logistic for binary variables, ordered logistic for ordered categories and linear for quantitative variables. Ordinal variables and quantitative variables were ordered in ascending order of severity. The relationship between LBP and occupational exposure to driving was estimated by multiple linear regression models with the latent variable as dependent variable, adjusting for individual factors, other occupational physical constraints and psychosocial factors, separately in men and women. All the factor loadings between the latent variable and LBP indicators were positive $(\mathrm{p}<0.001)$ indicating that the latent scale is consistent with health impairment on the low back. In men, driving time $(\mathrm{p}=0.007)$ and high perceived driving-related physical constraints $(p=0,024)$ were associated to LBP whereas in women no driving-related constraints were associated to LBP. In the absence of a standardized clinical tool, studies about association between occupational exposures and LBP could benefit from methods that are able to retrieve diffuse information broken out into ad hoc questionnaire.

\section{O4B.6 DO WE NEED REPEATED MEASUREMENTS FOR RELIABLE CLASSIFICATION OF CASE STATUS REGARDING MUSCULOSKELETAL PAIN?}

1,2 Lena Hillert*, ${ }^{2}$ Magnus Alderling, ${ }^{1,3}$ Carl Lind, ${ }^{1,2}$ Annika Lindahl Norberg,
1,2,3 Mikael Forsman. ${ }^{1}$ Karolinska Institutet, Stockholm, Sweden; ${ }^{2}$ Centre for Occupational
and Environmental Medicine, Stockholm County Council, Stockholm, Sweden; ${ }^{3}$ KTH Royal
Institute of Technology, Stockholm, Sweden

10.1136/OEM-2019-EPI.96

Musculoskeletal disorders (MSDs) are major causes for disability, reduced work ability and early exit from the labour market. The aetiology is understood to be multifactorial and MSDs are known to have a recurrent course characterized by development, recovery, reoccurrence and chronic states. Studies classifying participants as suffering from e.g. low back pain based on self-reported symptoms solely at one time are therefore at risk of just capturing the point prevalence of pain and not a more representative state characterizing the participants.

In order to investigate how well a classification based on self-reported musculoskeletal complaints of the neck, shoulders and lower back in a baseline questionnaire represents the situation of the respondents' pain and functional status over a 12 month period, we analysed data collected in a study on 129 employees at selected Swedish home-care providers. The participants completed a baseline questionnaire on work environment and mental and physical health and responded to questions on the impact on work capacity of pain sent monthly to their phones by text messages.

The results show that classification into cases and non-cases cases based on reported musculoskeletal complaints at baseline was a statistically significant predictor of persistent differences between groups with regard to pain and work capacity 